\title{
A Two-Stage Mutation Stochastic Model of Carcinogenesis Driven by a Three Level Environmental Process
}

\author{
V.S. S. Yadavalli \\ Department of Industrial and System Engineering, \\ University of Pretoria, Pretoria 0002, South Africa. \\ Corresponding author: sarma.yadavalli@up.ac.za \\ S. Udayabaskaran \\ Department of Mathematics, \\ Vel Tech Rangarajan Dr. Sagunthala R\&D Institute of Science and Technology, \\ Avadi, Chennai 600062, India. \\ E-mail: sudayabaskaran@veltech.edu.in \\ C. T. Dora Pravina \\ Department of Mathematics, \\ Vel Tech Rangarajan Dr. Sagunthala R\&D Institute of Science and Technology, \\ Avadi, Chennai 600062, India. \\ E-mail: tdorapravinac@ veltech.edu.in \\ S. Sreelakshmi \\ Department of Engineering Mathematics, \\ HKBK College of Engineering, Bangalore 560045, India. \\ E-mail: sreelakshmi.mt@hkbk.edu.in
}

(Received May 7, 2020; Accepted August 18, 2020)

\begin{abstract}
A two-mutation model of carcinogenesis which evolves under the influence of three level random environment on the production process is formulated and analyzed. A random environment occupies one of the levels 1, 2 and 3 at any time $t$ according to a Markov process. When the environment is in level 1, a normal cell either divides into two normal cells or dies; and an intermediate cell divides into two intermediate cells or dies. When the environment is in level 2 , a normal cell either divides into one normal cell and one intermediate cell or dies and an intermediate cell either divides into one intermediate cell and one malignant cell or dies. When the environment is in level 3, a normal cell either divides into two intermediate cells or dies and an intermediate cell either divides into two malignant cells or dies. It is assumed that, once a malignant cell is produced, it generates a malignant tumor with probability 1 . We obtain the mean numbers of normal, intermediate and malignant cells at any time $t$.
\end{abstract}

Keywords- Age-dependent two-stage stochastic model, Normal cell, Intermediate cell, Malignant cell, Random environment.

\section{Introduction}

Recently there have been an increased interest in the study of age-dependent/ environmentdependent models of carcinogenesis. Sun et al. (2014) have formulated a model of time scheme for progression of colorectal cancer based upon maturity and predicted the values of several important parameters in cancer progression. Tomasetti and Vogelstein (2015) have taken into consideration random mutations arising during DNA replication in normal, noncancerous stem cells and studied cancer risk for individuals. Rozhok and DeGregori (2016) have presented a theoretical study of 
International Journal of Mathematical, Engineering and Management Sciences

Vol. 5, No. 6, 1468-1487, 2020

https://doi.org/10.33889/IJMEMS.2020.5.6.109

age-dependent models of cancer risk. Rozhok et al. (2016) have applied stochastic Monte Carlo methods to explain the age-dependent incidence of cancer. Reddy et al. (2017) have observed that during every cell division, some rare events at the genome level such as DNA replication mistakes take place of which some are of no importance, while some have significance for escaping cell division control mechanisms. Martincorena et al. (2017) have concluded that cancer risk is dependent on the random errors occurring in normal cell replication, hereditary defects in critical genes, and environmental factors including exogenous agents and lifestyle. Hochberg and Noble (2017) have provided a framework for understanding how natural environmental variation and human activity impact cancer risk, with potential implications for species ecology. Simulation studies have been reported in the paper of Rozhok and DeGregori (2019) on a generalized theory of age-dependent carcinogenesis to demonstarte the impact of key somatic evolutionary parameters on the performance of Multistage Model of Carcinogenesis. Wolf et al. (2019) have presented an unified theory of carcinogenesis in which they have considered multi-stage carcinogenesis models to assess the carcinogenicity of chemicals for risk management and the public communication. In the above studies, cell division mechanism have either dependance on random mutations arising during DNA replication in normal cells or on environment or external agents such as chemical substances or living habits. Mutation dependent stochastic models of carcinogenesis have been studied in a series of papers (see Nordling, 1953; Armitage and Doll, 1954, 1957 and 1961; Knudson, 1971; Moolgavkar and Venzon, 1979; Moolgavkar and Knudson, 1981). However, environment dependent stochastic models of carcinogenesis have not been analytically studied very much in literature. As per the observation of Martincorena et al. (2017), numerous replication mistakes take place during every cell division and these mistakes might be due to environmental effects. The paper of Martincorena et al. (2017) has triggered us to model a cancerous environment as a multi-level and study its impact on mutational process in cell division mechanism. In a recent paper, Yadavalli et al. (2020) have analyzed a mutation-dependent stochastic model of carcinogenesis where mutation is inflenced by a two-level environmental process. As it is very much appropriate to consider the environmental process as a multi-level process, two-level for environment is only a stringent assumption. Accordingly, we propose and analyze in the present paper, a two-stage stochastic model of carcinogenesis driven by a three-level random environment. Here, the random environment occupies one of the levels 1,2 and 3 according to a Markov process.

When the environment is in level 1, a normal cell either divides into two normal cells with rate $L_{1}$ or dies with rate $D_{1}$; and an intermediate cell divides into two intermediate cells with rate $\alpha_{1}$ or dies with rate $\mu_{1}$. When the environment is in level 2, a normal cell either divides into one normal cell and one intermediate cell with rate $L_{2}$ or dies with rate $D_{2}$; and an intermediate cell either divides into one intermediate cell and one malignant cell with rate $\alpha_{2}$ or dies with rate $\mu_{2}$. When the environment is in level 3, a normal cell either divides into two intermediate cells with rate $L_{3}$ or dies with rate $D_{3}$; and an intermediate cell either divides into two malignant cells with rate $\alpha_{3}$ or dies with rate $\mu_{3}$. It is assumed that, once a malignant cell is produced, it generates a malignant tumor with probability 1 . For this model, we obtain the mean numbers of normal, intermediate and malignant cells.

The paper is organized as follows: In section 2, we describe the random environment and formulate the two-stage mutation model of carcinogenesis driven by the random environment. In section 3, we write the integral equations satisfied by the conditional probability generating functions of the number of normal, intermediate and malignant cells. Section 4 obtains the mean number of normal, intermediate and malignant cells in the population. A numerical illustration is 
International Journal of Mathematical, Engineering and Management Sciences

Vol. 5, No. 6, 1468-1487, 2020

https://doi.org/10.33889/IJMEMS.2020.5.6.109

provided in Section 5 and a comparison is made with the results of Yadavalli et al. (2020). A conclusion is presented in Section 6.

\section{Model Formulation}

We consider a cell population consisting of normal, intermediate and malignant cells. Let $X(t), Y(t)$ and $Z(t)$ be the random variables denoting the number of normal, intermediate and malignant cells existing at time $t$. Let $\eta(t)$ denote the state of the environment at time $t$. We call the time duration a cell has lived without splitting since its birth time as the age of the cell.

\subsection{Assumptions}

(i) All cells evolve in a cancerous random environment.

(ii) At any time $t$, the random environment is in one of the three levels 1, 2 and 3.

(iii) $\quad P[\eta(t+\Delta t)=2 \mid \eta(t)=1]=\gamma_{12} \Delta t+o(\Delta t)$

(iv) $\quad P[\eta(t+\Delta t)=3 \mid \eta(t)=1]=\gamma_{13} \Delta t+o(\Delta t)$

(v) $\quad P[\eta(t+\Delta t)=1 \mid \eta(t)=1]=1-\left(\gamma_{12}+\gamma_{13}\right) \Delta t+o(\Delta t)$

(vi) $\quad P[\eta(t+\Delta t)=3 \mid \eta(t)=2]=\gamma_{23} \Delta t+o(\Delta t)$

(vii) $\quad P[\eta(t+\Delta t)=1 \mid \eta(t)=2]=\gamma_{21} \Delta t+o(\Delta t)$

(viii) $\quad P[\eta(t+\Delta t)=2 \mid \eta(t)=2]=1-\left(\gamma_{23}+\gamma_{21}\right) \Delta t+o(\Delta t)$

(ix) $\quad P[\eta(t+\Delta t)=1 \mid \eta(t)=3]=\gamma_{31} \Delta t+o(\Delta t)$

(x) $\quad P[\eta(t+\Delta t)=2 \mid \eta(t)=3]=\gamma_{32} \Delta t+o(\Delta t)$

(xi) $\quad P[\eta(t+\Delta t)=3 \mid \eta(t)=3]=1-\left(\gamma_{31}+\gamma_{32}\right) \Delta t+o(\Delta t)$

(xii) When the environment is in level 1 at time $t$, a normal cell existing at time $t$ either divides into two normal cells with probability $L_{1} \Delta t+o(\Delta t)$ or dies with probability $D_{1} \Delta t+o(\Delta t)$ in an infinitesimal interval $(t, t+\Delta t)$.

(xiii) When the environment is in level 1 at time $t$, an intermediate cell existing at time $t$ divides into two intermediate cells with probability $\alpha_{1} \Delta t+o(\Delta t)$ or dies with probability $\mu_{1} \Delta t+o(\Delta t)$ in an infinitesimal interval $(t, t+\Delta t)$.

(xiv) When the environment is in level 2 at time $t$, a normal cell existing at time $t$ either divides into one normal cell and one intermediate cell with probability $L_{2} \Delta t+o(\Delta t)$ or dies with probability $D_{2} \Delta t+o(\Delta t)$ in an infinitesimal interval $(t, t+\Delta t)$.

(xv) When the environment is in level 2 at time $t$, an intermediate cell existing at time $t$ either divides into one intermediate cell and one malignant cell with probability $\alpha_{2} \Delta t+o(\Delta t)$ or dies with probability $\mu_{2} \Delta t+o(\Delta t)$ in an infinitesimal interval $(t, t+$ $\Delta t)$.

(xvi) When the environment is in state 3 at time $t$, a normal cell existing at time $t$ either divides into two intermediate cells with probability $L_{3} \Delta t+o(\Delta t)$ or dies with probability $D_{3} \Delta t+o(\Delta t)$ in an infinitesimal interval $(t, t+\Delta t)$.

(xvii) When the environment is in level 3 at time $t$, an intermediate cell existing at time $t$ either two malignant cells with probability $\alpha_{3} \Delta t+o(\Delta t)$ or dies with Probability $\mu_{3} \Delta t+o(\Delta t)$ in an infinitesimal interval $(t, t+\Delta t)$.

(xviii) Once a malignant cell is produced, it generates a malignant tumor with probability 1 .

(xix) All events are independent and the probability of occurrence of more than one event in a small interval $(t, t+\Delta t)$ is $o(\Delta t)$.

\section{Governing Equations}

We define the conditional probability generating functions for the number of normal, intermediate and malignant cells at time $t$ as follows: 
International Journal of Mathematical, Engineering and Management Sciences

Vol. 5, No. 6, 1468-1487, 2020

https://doi.org/10.33889/IJMEMS.2020.5.6.109

$$
\begin{gathered}
\psi_{j}(x, y, z, t)=E\left[x^{X(t)} y^{Y(t)} z^{Z(t)} \mid X(0)=1, Y(0)=0, Z(0)=0, \eta(0)=j\right], j=1,2,3 ; \\
\phi_{j}(y, z, t)=E\left[y^{Y(t)} z^{Z(t)} \mid Y(0)=1, Z(0)=0, \eta(0)=j\right], j=1,2,3 .
\end{gathered}
$$

In what follows, we hide the variables $x, y$ and $z$ in the functions $\phi_{j}$ and $\psi_{j}$, and simply write $\phi_{j}(t)$ and $\psi_{j}(t)$ unless otherwise needed. The function $\phi_{j}(t), j=1,2,3$ correspond to onemutation model, and $\psi_{j}(t), j=1,2,3$ correspond to two-mutation model. In the above definition, the condition $Y(0)=1, Z(0)=0$ means that we are starting with just one intermediate cell at time $t=0$. It is to be noted that intermediate cells do not contribute to the production of normal cells.

By the formulation of the model, the four-dimensional stochastic process $\{(X(t), Y(t), Z(t), \eta(t) \mid t \geq 0\}$ is Markov. The state-transition diagram is given in Figure 1 below:
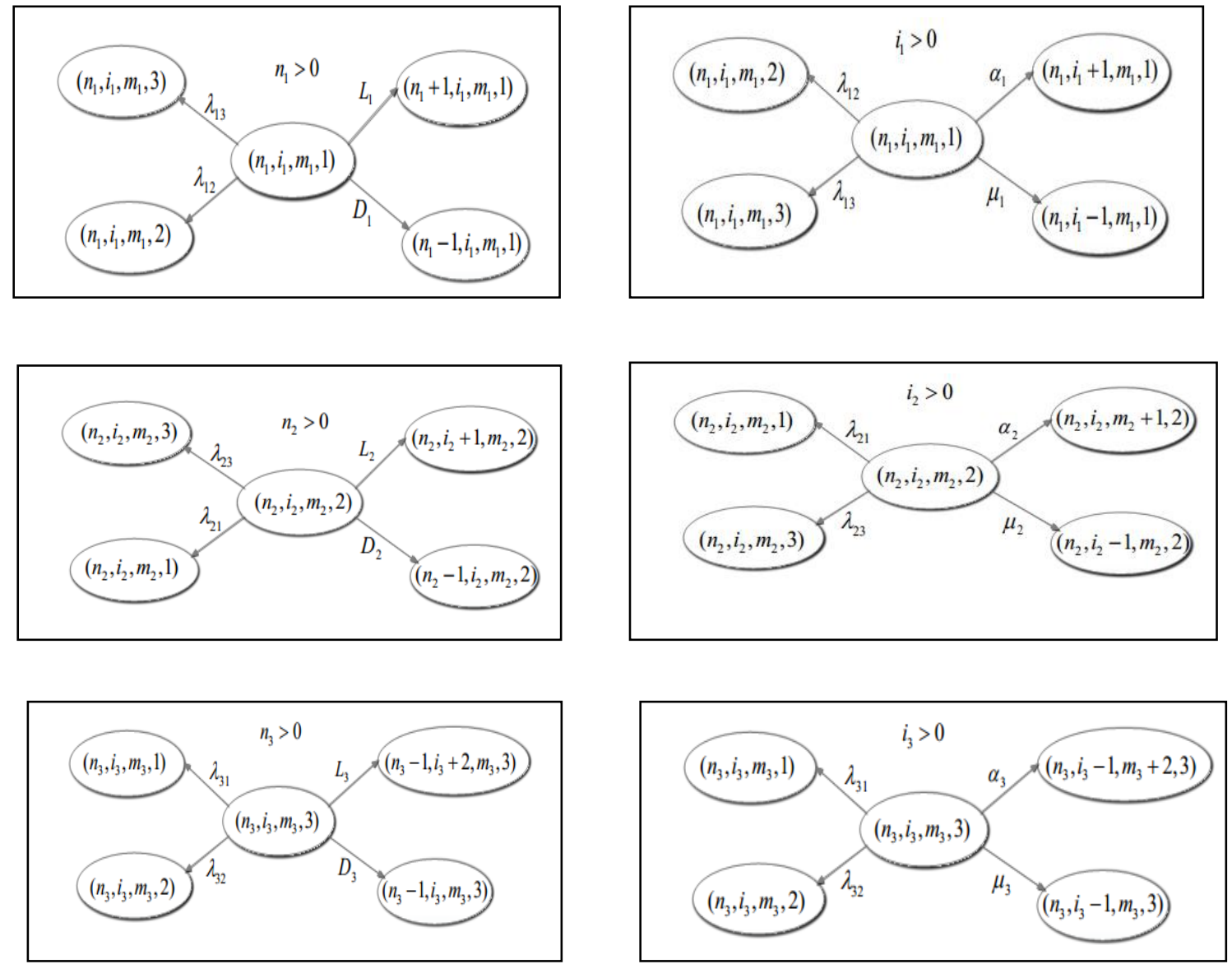

Figure 1. State transition diagram

Considering the first event happening in the time interval $(0, t)$ and using Bellmann's invariant 
International Journal of Mathematical, Engineering and Management Sciences

Vol. 5, No. 6, 1468-1487, 2020

https://doi.org/10.33889/IJMEMS.2020.5.6.109

imbedding technique (see Bellmann et al., 1960), we obtain the following integral equations for $\phi_{j}(t)$ and $\psi_{j}(t)$ :

$$
\begin{aligned}
\psi_{1}(t)= & x e^{-a_{1} t}+D_{1} \int_{0}^{t} e^{-a_{1} u} d u+L_{1} \int_{0}^{t} e^{-a_{1} u}\left\{\psi_{1}(t-u)\right\}^{2} d u+\gamma_{12} \int_{0}^{t} e^{-a_{1} u} \psi_{2}(t-u) d u+ \\
& \gamma_{13} \int_{0}^{t} e^{-a_{1} u} \psi_{3}(t-u) d u \\
\psi_{2}(t)= & x e^{-a_{2} t}+D_{2} \int_{0}^{t} e^{-a_{2} u} d u+L_{2} \int_{0}^{t} e^{-a_{2} u} \psi_{2}(t-u) \phi_{2}(t-u) d u+\gamma_{21} \int_{0}^{t} e^{-a_{2} u} \psi_{1}(t- \\
& u) d u+\gamma_{23} \int_{0}^{t} e^{-a_{2} u} \psi_{3}(t-u) d u
\end{aligned}
$$

where,

$$
\begin{gathered}
a_{1}=\gamma_{12}+\gamma_{13}+L_{1}+D_{1} ; a_{2}=\gamma_{23}+\gamma_{21}+L_{2}+D_{2} ; a_{3}=\gamma_{31}+\gamma_{32}+L_{3}+D_{3} \\
b_{1}=\gamma_{12}+\gamma_{13}+\alpha_{1}+\mu_{1} ; b_{2}=\gamma_{23}+\gamma_{21}+\alpha_{2}+\mu_{2} ; b_{3}=\gamma_{31}+\gamma_{32}+\alpha_{3}+\mu_{3}
\end{gathered}
$$

\section{Mean Numbers of Cells}

We consider the following conditional means:

$$
\begin{array}{crrr}
m_{X, j}{ }^{(2)}(t)=E[X(t) \mid X(0)=1, Y(0)=0, Z(0)=0, \eta(0)=j], & & j=1,2,3 ; \\
m_{Y, j}{ }^{(2)}(t)=E[Y(t) \mid X(0)=1, Y(0)=0, Z(0)=0, \eta(0)=j], & j=1,2,3 ; \\
m_{Z, j}{ }^{(2)}(t)=E[Z(t) \mid X(0)=1, Y(0)=0, Z(0)=0, \eta(0)=j], & j=1,2,3 ; \\
m_{Y, j}{ }^{(1)}(t)=E[Y(t) \mid Y(0)=1, Z(0)=0, \eta(0)=j] & j=1,2,3 ; \\
m_{Z, j}{ }^{(1)}(t)=E[Z(t) \mid Y(0)=1, Z(0)=0, \eta(0)=j] & j=1,2,3 .
\end{array}
$$


International Journal of Mathematical, Engineering and Management Sciences

Vol. 5, No. 6, 1468-1487, 2020

https://doi.org/10.33889/IJMEMS.2020.5.6.109

From the definitions of $\psi_{j}(t)$ and $\phi_{j}(t)$, we get

$$
\begin{gathered}
m_{X, j}{ }^{(2)}(t)=\left[\frac{\partial \psi_{j}(t)}{\partial x}\right]_{x=1, y=1, z=1}, m_{Y, j}{ }^{(2)}(t)=\left[\frac{\partial \psi_{j}(t)}{\partial y}\right]_{x=1, y=1, z=1} \\
m_{Z, j}{ }^{(2)}(t)=\left[\frac{\partial \psi_{j}(t)}{\partial z}\right]_{x=1, y=1, z=1}, \\
m_{Y, j}{ }^{(1)}(t)=\left[\frac{\partial \phi_{j}(t)}{\partial y}\right]_{y=1, z=1}, m_{Z, j}^{(1)}(t)=\left[\frac{\partial \phi_{j}(t)}{\partial z}\right]_{y=1, z=1} .
\end{gathered}
$$

Differentiating (1), (2) and (3) partially with respect to $x$, and putting $x=1, y=1$ and $z=1$, we get respectively

$$
\begin{aligned}
m_{X, 1}{ }^{(2)}(t)= & e^{-a_{1} t}+2 L_{1} \int_{0}^{t} e^{-a_{1} u} m_{X, 1}{ }^{(2)}(t-u) d u+\gamma_{12} \int_{0}^{t} e^{-a_{1} u} m_{X, 2}{ }^{(2)}(t-u) d u+ \\
& \gamma_{13} \int_{0}^{t} e^{-a_{1} u} m_{X, 3}{ }^{(2)}(t-u) d u \\
m_{X, 2}{ }^{(2)}(t)= & e^{-a_{2} t}+L_{2} \int_{0}^{t} e^{-a_{2} u} m_{X, 2}{ }^{(2)}(t-u) d u+\gamma_{21} \int_{0}^{t} e^{-a_{2} u} m_{X, 1}{ }^{(2)}(t-u) d u+ \\
& \gamma_{23} \int_{0}^{t} e^{-a_{2} u} m_{X, 3}{ }^{(2)}(t-u) d u \\
m_{X, 3}{ }^{(2)}(t)= & e^{-a_{3} t}+\gamma_{31} \int_{0}^{t} e^{-a_{3} u} m_{X, 1}{ }^{(2)}(t-u) d u+\gamma_{32} \int_{0}^{t} e^{-a_{3} u} m_{X, 2}{ }^{(2)}(t-u) d u .
\end{aligned}
$$

Differentiating (1), (2) and (3) partially with respect to $y$, and putting $x=1, y=1$ and $z=1$, we get respectively

$$
\begin{aligned}
& m_{Y, 1}{ }^{(2)}(t)= 2 L_{1} \int_{0}^{t} e^{-a_{1} u} m_{Y, 1}{ }^{(2)}(t-u) d u+\gamma_{12} \int_{0}^{t} e^{-a_{1} u} m_{Y, 2}{ }^{(2)}(t-u) d u+ \\
& \gamma_{13} \int_{0}^{t} e^{-a_{1} u} m_{Y, 3}{ }^{(2)}(t-u) d u \\
& m_{Y, 2}{ }^{(2)}(t)= L_{2} \int_{0}^{t} e^{-a_{2} u}\left[m_{Y, 2}{ }^{(2)}(t-u)+m_{Y, 2}{ }^{(1)}(t-u)\right] d u+\gamma_{21} \int_{0}^{t} e^{-a_{2} u} m_{Y, 1}{ }^{(2)}(t- \\
&u) d u+\gamma_{23} \int_{0}^{t} e^{-a_{2} u} m_{Y, 3}{ }^{(2)}(t-u) d u \\
& m_{Y, 3}{ }^{(2)}(t)= 2 L_{3} \int_{0}^{t} e^{-a_{3} u} m_{Y, 3}^{(1)}(t-u) d u+\gamma_{31} \int_{0}^{t} e^{-a_{3} u} m_{Y, 1}{ }^{(2)}(t-u) d u+ \\
& \gamma_{32} \int_{0}^{t} e^{-a_{3} u} m_{Y, 2}{ }^{(2)}(t-u) d u .
\end{aligned}
$$

Differentiating (1), (2) and (3) partially with respect to $z$, and putting $x=1, y=1$ and $z=1$, we get respectively

$$
\begin{aligned}
m_{Z, 1}{ }^{(2)}(t)= & 2 L_{1} \int_{0}^{t} e^{-a_{1} u} m_{Z, 1}{ }^{(2)}(t-u) d u+\gamma_{12} \int_{0}^{t} e^{-a_{1} u} m_{Z, 2}{ }^{(2)}(t-u) d u+ \\
& \gamma_{13} \int_{0}^{t} e^{-a_{1} u} m_{Z, 3}{ }^{(2)}(t-u) d u
\end{aligned}
$$


International Journal of Mathematical, Engineering and Management Sciences

Vol. 5, No. 6, 1468-1487, 2020

https://doi.org/10.33889/IJMEMS.2020.5.6.109

$$
\begin{array}{rl}
m_{Z, 2}{ }^{(2)}(t)= & L_{2} \int_{0}^{t} e^{-a_{2} u}\left[m_{Z, 2}{ }^{(2)}(t-u)+m_{Z, 2}{ }^{(1)}(t-u)\right] d u+\gamma_{21} \int_{0}^{t} e^{-a_{2} u} m_{Z, 1}{ }^{(2)}(t- \\
u & d u+\gamma_{23} \int_{0}^{t} e^{-a_{2} u} m_{Z, 3}{ }^{(2)}(t-u) d u \\
m_{Z, 3}{ }^{(2)}(t)= & 2 L_{3} \int_{0}^{t} e^{-a_{3} u} m_{Z, 3}{ }^{(1)}(t-u) d u+\gamma_{31} \int_{0}^{t} e^{-a_{3} u} m_{Z, 1}{ }^{(2)}(t-u) d u+ \\
& \gamma_{32} \int_{0}^{t} e^{-a_{3} u} m_{Z, 2}{ }^{(2)}(t-u) d u .
\end{array}
$$

Differentiating (4), (5) and (6) partially with respect to $y$, and putting $y=1$ and $z=1$, we get respectively

$$
\begin{aligned}
m_{Y, 1}{ }^{(1)}(t)= & e^{-b_{1} t}+2 \alpha_{1} \int_{0}^{t} e^{-b_{1} u} m_{Y, 1}{ }^{(1)}(t-u) d u+\gamma_{12} \int_{0}^{t} e^{-b_{1} u} m_{Y, 2}{ }^{(1)}(t-u) d u+ \\
& \gamma_{13} \int_{0}^{t} e^{-b_{1} u} m_{Y, 3}{ }^{(1)}(t-u) d u, \\
m_{Y, 2}{ }^{(1)}(t)= & e^{-b_{2} t}+\alpha_{2} \int_{0}^{t} e^{-b_{2} u} m_{Y, 2}{ }^{(1)}(t-u) d u+\gamma_{21} \int_{0}^{t} e^{-b_{2} u} m_{Y, 1}{ }^{(1)}(t-u) d u+ \\
& \gamma_{23} \int_{0}^{t} e^{-b_{2} u} m_{Y, 3}{ }^{(1)}(t-u) d u \\
m_{Y, 3}{ }^{(1)}(t)= & e^{-b_{3} t}+\gamma_{31} \int_{0}^{t} e^{-b_{3} u} m_{Y, 1}{ }^{(1)}(t-u) d u+\gamma_{32} \int_{0}^{t} e^{-b_{3} u} m_{Y, 2}{ }^{(1)}(t-u) d u
\end{aligned}
$$

Differentiating (4), (5) and (6) partially with respect to $z$, and putting $y=1$ and $z=1$, we get respectively

$$
\begin{aligned}
m_{Z, 1}{ }^{(1)}(t)= & 2 \alpha_{1} \int_{0}^{t} e^{-b_{1} u} m_{Z, 1}{ }^{(1)}(t-u) d u+\gamma_{12} \int_{0}^{t} e^{-b_{1} u} m_{Z, 2}{ }^{(1)}(t-u) d u+ \\
& \gamma_{13} \int_{0}^{t} e^{-b_{1} u} m_{Z, 3}{ }^{(1)}(t-u) d u \\
m_{Z, 2}{ }^{(1)}(t)= & \alpha_{2} \int_{0}^{t} e^{-b_{2} u} d u+\alpha_{2} \int_{0}^{t} e^{-b_{2} u} m_{Z, 2}{ }^{(1)}(t-u) d u+\gamma_{21} \int_{0}^{t} e^{-b_{2} u} m_{Z, 1}{ }^{(1)}(t-u) d u+ \\
& \gamma_{23} \int_{0}^{t} e^{-b_{2} u} m_{Z, 3}{ }^{(1)}(t-u) d u \\
m_{Z, 3}{ }^{(1)}(t)= & 2 \alpha_{3} \int_{0}^{t} e^{-b_{3} u} d u+\gamma_{31} \int_{0}^{t} e^{-b_{3} u} m_{Z, 1}{ }^{(1)}(t-u) d u+ \\
& \gamma_{32} \int_{0}^{t} e^{-b_{3} u} m_{Z, 2}{ }^{(1)}(t-u) d u
\end{aligned}
$$

The system of integral equations (7) - (21) are inter-connected. Taking Laplace transform on both sides of (19), (20) and (21), we get

$$
\begin{aligned}
& {\left[\left(s+b_{1}\right)-2 \alpha_{1}\right] m_{Z, 1}{ }^{(1) *}(s)-\gamma_{12} m_{Z, 2}{ }^{(1) *}(s)-\gamma_{13} m_{Z, 3}{ }^{(1) *}(s)=0,} \\
& -\gamma_{21} m_{Z, 1}{ }^{(1) *}(s)+\left[(s+b 2)-\alpha_{2}\right] m_{Z, 2}{ }^{(1) *}(s)-\gamma_{23} m_{Z, 3}{ }^{(1) *}(s)=\frac{\alpha_{2}}{s},
\end{aligned}
$$


International Journal of Mathematical, Engineering and Management Sciences

Vol. 5, No. 6, 1468-1487, 2020

https://doi.org/10.33889/IJMEMS.2020.5.6.109

$-\gamma_{31} m_{Z, 1}{ }^{(1) *}(s)-\gamma_{32} m_{Z, 2}{ }^{(1) *}(s)+\left(s+b_{3}\right) m_{Z, 3}{ }^{(1) *}(s)=\frac{2 \alpha_{3}}{s}$.

Solving (22), (23) and (24), we get

$m_{Z, 1}{ }^{(1) *}(s)=\frac{\Delta_{31}(s)}{s \Delta(s)}, m_{Z, 2}{ }^{(1) *}(s)=\frac{\Delta_{32}(s)}{s \Delta(s)}, m_{Z, 3}{ }^{(1) *}(s)=\frac{\Delta_{33}(s)}{s \Delta(s)}$,

where,

$\Delta(s)=\left|\begin{array}{ccc}s+b_{1}-2 \alpha_{1} & -\gamma_{12} & -\gamma_{13} \\ -\gamma_{21} & s+b_{2}-\alpha_{2} & -\gamma_{23} \\ -\gamma_{31} & -\gamma_{32} & s+b_{3}\end{array}\right|$,

$\Delta_{31}(s)=\left|\begin{array}{ccc}0 & -\gamma_{12} & -\gamma_{13} \\ \alpha_{2} & s+b_{2}-\alpha_{2} & -\gamma_{23} \\ 2 \alpha_{3} & -\gamma_{32} & s+b_{3}\end{array}\right|$,

$\Delta_{32}(s)=\left|\begin{array}{ccc}s+b_{1}-2 \alpha_{1} & 0 & -\gamma_{13} \\ -\gamma_{21} & \alpha_{2} & -\gamma_{23} \\ -\gamma_{31} & 2 \alpha_{3} & s+b_{3}\end{array}\right|$,

$\Delta_{33}(s)=\left|\begin{array}{ccc}s+b_{1}-2 \alpha_{1} & -\gamma_{12} & 0 \\ -\gamma_{21} & s+b_{2}-\alpha_{2} & \alpha_{2} \\ -\gamma_{31} & -\gamma_{32} & 2 \alpha_{3}\end{array}\right|$.

$m_{Z, 3}{ }^{(1) *}(s)=\frac{\Delta_{33}(s)}{s\left(s-\omega_{1}\right)\left(s-\omega_{2}\right)\left(s-\omega_{3}\right)}$.

Splitting into partial fractions, and then taking inverse Laplace transform, equations (30) - (32) yield

$$
\begin{aligned}
m_{Z, 1}{ }^{(1)}(t)= & -\frac{1}{\omega_{1} \omega_{2} \omega_{3}}\left[\Delta_{31}(0)+\frac{\omega_{2} \omega_{3} \Delta_{31}\left(\omega_{1}\right)}{\left(\omega_{1}-\omega_{2}\right)\left(\omega_{3}-\omega_{1}\right)} e^{\omega_{1} t}+\frac{\omega_{3} \omega_{1} \Delta_{31}\left(\omega_{2}\right)}{\left(\omega_{1}-\omega_{2}\right)\left(\omega_{2}-\omega_{3}\right)} e^{\omega_{2} t}+\right. \\
& \left.\frac{\omega_{1} \omega_{2} \Delta_{31}\left(\omega_{3}\right)}{\left(\omega_{3}-\omega_{1}\right)\left(\omega_{2}-\omega_{3}\right)} e^{\omega_{3} t}\right], \\
m_{Z, 2}{ }^{(1)}(t)= & -\frac{1}{\omega_{1} \omega_{2} \omega_{3}}\left[\Delta_{32}(0)+\frac{\omega_{2} \omega_{3} \Delta_{32}\left(\omega_{1}\right)}{\left(\omega_{1}-\omega_{2}\right)\left(\omega_{3}-\omega_{1}\right)} e^{\omega_{1} t}+\frac{\omega_{3} \omega_{1} \Delta_{32}\left(\omega_{2}\right)}{\left(\omega_{1}-\omega_{2}\right)\left(\omega_{2}-\omega_{3}\right)} e^{\omega_{2} t}+\right. \\
& \left.\frac{\omega_{1} \omega_{2} \Delta_{32}\left(\omega_{3}\right)}{\left(\omega_{3}-\omega_{1}\right)\left(\omega_{2}-\omega_{3}\right)} e^{\omega_{3} t}\right]
\end{aligned}
$$


International Journal of Mathematical, Engineering and Management Sciences

Vol. 5, No. 6, 1468-1487, 2020

https://doi.org/10.33889/IJMEMS.2020.5.6.109

$$
\begin{aligned}
m_{Z, 3}{ }^{(1)}(t)= & -\frac{1}{\omega_{1} \omega_{2} \omega_{3}}\left[\Delta_{33}(0)+\frac{\omega_{2} \omega_{3} \Delta_{33}\left(\omega_{1}\right)}{\left(\omega_{1}-\omega_{2}\right)\left(\omega_{3}-\omega_{1}\right)} e^{\omega_{1} t}+\frac{\omega_{3} \omega_{1} \Delta_{33}\left(\omega_{2}\right)}{\left(\omega_{1}-\omega_{2}\right)\left(\omega_{2}-\omega_{3}\right)} e^{\omega_{2} t}+\right. \\
& \left.\frac{\omega_{1} \omega_{2} \Delta_{33}\left(\omega_{3}\right)}{\left(\omega_{3}-\omega_{1}\right)\left(\omega_{2}-\omega_{3}\right)} e^{\omega_{3} t}\right] .
\end{aligned}
$$

Taking Laplace transform on both sides of (16), (17) and (18), we get

$$
\begin{aligned}
& \left(s+b_{1}-2 \alpha_{1}\right) m_{Y, 1}{ }^{(1) *}(s)-\gamma_{12} m_{Y, 2}{ }^{(1) *}(s)-\gamma_{13} m_{Y, 3}{ }^{(1) *}(s)=1, \\
& -\gamma_{21} m_{Y, 1}{ }^{(1) *}(s)+\left(s+b_{2}-\alpha_{2}\right) m_{Y, 2}{ }^{(1) *}(s)-\gamma_{23} m_{Y, 3}{ }^{(1) *}(s)=1, \\
& -\gamma_{31} m_{Y, 1}{ }^{(1) *}(s)-\gamma_{32} m_{Y, 2}{ }^{(1) *}(s)+\left(s+b_{3}\right) m_{Y, 3}{ }^{(1) *}(s)=1 .
\end{aligned}
$$

Solving (36), (37) and (38), we get

$$
m_{Y, 1}^{(1) *}(s)=\frac{\Delta_{21}(s)}{\Delta(s)}, m_{Y, 2}^{(1) *}(s)=\frac{\Delta_{22}(s)}{\Delta(s)}, m_{Y, 3}^{(1) *}(s)=\frac{\Delta_{23}(s)}{\Delta(s)} .
$$

where, $\Delta(s)$ is given by (26), and

$$
\begin{aligned}
& \Delta_{21}(s)=\left|\begin{array}{ccc}
1 & -\gamma_{12} & -\gamma_{13} \\
1 & s+b_{2}-\alpha_{2} & -\gamma_{23} \\
1 & -\gamma_{32} & s+b_{3}
\end{array}\right|, \\
& \Delta_{22}(s)=\left|\begin{array}{ccc}
s+b_{1}-2 \alpha_{1} & 1 & -\gamma_{13} \\
-\gamma_{21} & 1 & -\gamma_{23} \\
-\gamma_{31} & 1 & s+b_{3}
\end{array}\right|, \\
& \Delta_{23}(s)=\left|\begin{array}{ccc}
s+b_{1}-2 \alpha_{1} & -\gamma_{12} & 1 \\
-\gamma_{21} & s+b_{2}-\alpha_{2} & 1 \\
-\gamma_{31} & -\gamma_{32} & 1
\end{array}\right| .
\end{aligned}
$$

Since $\Delta(s)=\left(s-\omega_{1}\right)\left(s-\omega_{2}\right)\left(s-\omega_{3}\right)$, equations in (39) yield

$$
\begin{aligned}
& m_{Y, 1}^{(1) *}(s)=\frac{\Delta_{21}(s)}{\left(s-\omega_{1}\right)\left(s-\omega_{2}\right)\left(s-\omega_{3}\right)}, \\
& m_{Y, 2}^{(1) *}(s)=\frac{\Delta_{22}(s)}{\left(s-\omega_{1}\right)\left(s-\omega_{2}\right)\left(s-\omega_{3}\right)}, \\
& m_{Y, 3}^{(1) *}(s)=\frac{\Delta_{23}(s)}{\left(s-\omega_{1}\right)\left(s-\omega_{2}\right)\left(s-\omega_{3}\right)} .
\end{aligned}
$$

Splitting into partial fractions, and then taking inverse Laplace transform, (43), (44) and (45) give

$$
\begin{aligned}
& m_{Y, 1}^{(1)}(t)=\frac{\Delta_{21}\left(\omega_{1}\right)}{\left(\omega_{1}-\omega_{2}\right)\left(\omega_{1}-\omega_{3}\right)} e^{\omega_{1} t}+\frac{\Delta_{21}\left(\omega_{2}\right)}{\left(\omega_{2}-\omega_{1}\right)\left(\omega_{2}-\omega_{3}\right)} e^{\omega_{2} t}+\frac{\Delta_{21}\left(\omega_{3}\right)}{\left(\omega_{3}-\omega_{1}\right)\left(\omega_{3}-\omega_{2}\right)} e^{\omega_{3} t}, \\
& m_{Y, 2}^{(1)}(t)=\frac{\Delta_{22}\left(\omega_{1}\right)}{\left(\omega_{1}-\omega_{2}\right)\left(\omega_{1}-\omega_{3}\right)} e^{\omega_{1} t}+\frac{\Delta_{22}\left(\omega_{2}\right)}{\left(\omega_{2}-\omega_{1}\right)\left(\omega_{2}-\omega_{3}\right)} e^{\omega_{2} t}+\frac{\Delta_{22}\left(\omega_{3}\right)}{\left(\omega_{3}-\omega_{1}\right)\left(\omega_{3}-\omega_{2}\right)} e^{\omega_{3} t},
\end{aligned}
$$


International Journal of Mathematical, Engineering and Management Sciences

Vol. 5, No. 6, 1468-1487, 2020

https://doi.org/10.33889/IJMEMS.2020.5.6.109

$m_{Y, 3}^{(1)}(t)=\frac{\Delta_{23}\left(\omega_{1}\right)}{\left(\omega_{1}-\omega_{2}\right)\left(\omega_{1}-\omega_{3}\right)} e^{\omega_{1} t}+\frac{\Delta_{23}\left(\omega_{2}\right)}{\left(\omega_{2}-\omega_{1}\right)\left(\omega_{2}-\omega_{3}\right)} e^{\omega_{2} t}+\frac{\Delta_{23}\left(\omega_{3}\right)}{\left(\omega_{3}-\omega_{1}\right)\left(\omega_{3}-\omega_{2}\right)} e^{\omega_{3} t}$.

Taking Laplace transform on both sides of (13), (14) and (15), we get

$$
\begin{aligned}
& \left(s+a_{1}-2 L_{1}\right) m_{Z, 1}^{(2) *}(s)-\gamma_{12} m_{Z, 2}^{(2) *}(s)-\gamma_{13} m_{Z, 3}^{(2) *}(s)=0, \\
& -\gamma_{21} m_{Z, 1}^{(2) *}(s)+\left(s+a_{2}-L_{2}\right) m_{Z, 2}^{(2) *}(s)-\gamma_{23} m_{Z, 3}^{(2) *}(s)=L_{2} m_{Z, 2}^{(1) *}(s), \\
& -\gamma_{31} m_{Z, 1}^{(2) *}(s)-\gamma_{32} m_{Z, 2}^{(2) *}(s)+\left(s+a_{3}\right) m_{Z, 3}^{(2) *}(s)=2 L_{3} m_{Z, 3}^{(1) *}(s) .
\end{aligned}
$$

Solving (49),(50) and (51), we get

$m_{Z, 1}^{(2) *}(s)=\frac{\Pi_{31}(s)}{\Pi(s)}, m_{Z, 2}^{(2) *}(s)=\frac{\Pi_{32}(s)}{\Pi(s)}, m_{Z, 3}^{(2) *}(s)=\frac{\Pi_{33}(s)}{\Pi(s)}$,

where,

$$
\begin{aligned}
& \Pi(s)=\left|\begin{array}{ccc}
s+a_{1}-2 L_{1} & -\gamma_{12} & -\gamma_{13} \\
-\gamma_{21} & s+a_{2}-L_{2} & -\gamma_{23} \\
-\gamma_{31} & -\gamma_{32} & s+a_{3}
\end{array}\right|, \\
& \Pi_{31}(s)=\left|\begin{array}{ccc}
0 & -\gamma_{12} & -\gamma_{13} \\
L_{2} m_{Z, 2}^{(1) *}(s) & s+a_{2}-L_{2} & -\gamma_{23} \\
2 L_{3} m_{Z, 3}^{(1) *}(s) & -\gamma_{32} & s+a_{3}
\end{array}\right|, \\
& \Pi_{32}(s)=\left|\begin{array}{ccc}
s+a_{1}-2 L_{1} & 0 & -\gamma_{13} \\
-\gamma_{21} & L_{2} m_{Z, 2}^{(1) *}(s) & -\gamma_{23} \\
-\gamma_{31} & 2 L_{3} m_{Z, 3}^{(1) *}(s) & s+a_{3}
\end{array}\right|, \\
& \Pi_{33}(s)=\left|\begin{array}{ccc}
s+a_{1}-2 L_{1} & -\gamma_{12} & 0 \\
-\gamma_{21} & s+a_{2}-L_{2} & L_{2} m_{Z, 2}^{(1) *}(s) \\
-\gamma_{31} & -\gamma_{32} & 2 L_{3} m_{Z, 3}^{(1) *}(s)
\end{array}\right| .
\end{aligned}
$$

Substituting (31) and (32) in (54),(55) and (56) and simplifying, we get

$$
\begin{aligned}
& \Pi_{31}(s)=\frac{p_{31}(s)}{s\left(s-\omega_{1}\right)\left(s-\omega_{2}\right)\left(s-\omega_{3}\right)}, \\
& \Pi_{32}(s)=\frac{p_{32}(s)}{s\left(s-\omega_{1}\right)\left(s-\omega_{2}\right)\left(s-\omega_{3}\right)}, \\
& \Pi_{33}(s)=\frac{p_{33}(s)}{s\left(s-\omega_{1}\right)\left(s-\omega_{2}\right)\left(s-\omega_{3}\right)},
\end{aligned}
$$

where, 
International Journal of Mathematical, Engineering and Management Sciences

Vol. 5, No. 6, 1468-1487, 2020

https://doi.org/10.33889/IJMEMS.2020.5.6.109

$$
\begin{aligned}
p_{31}(s)= & L_{2} \Delta_{32}(s)\left[\gamma_{12}\left(s+a_{3}\right)+\gamma_{13} \gamma_{32}\right]+2 L_{3} \Delta_{33}(s)\left[\gamma_{12} \gamma_{23}+\gamma_{13}\left(s+a_{2}-L_{2}\right)\right], \\
p_{32}(s)= & L_{2} \Delta_{32}(s)\left[\left(s+a_{1}-2 L_{1}\right)\left(s+a_{3}\right)-\gamma_{13} \gamma_{31}\right]+2 L_{3} \Delta_{33}(s)\left[\gamma _ { 2 3 } \left(s+a_{1}-2 L_{1}\right.\right. \\
& \left.+\gamma_{13} \gamma_{21}\right], \\
p_{33}(s)= & L_{2} \Delta_{32}(s)\left[\gamma_{32}\left(s+a_{1}-2 L_{1}\right)+\gamma_{12} \gamma_{31}\right]+2 L_{3} \Delta_{33}(s)\left[\left(s+a_{1}-2 L_{1}\right)\left(s+a_{2}-L_{2}\right)\right. \\
& \left.-\gamma_{12} \gamma_{21}\right] .
\end{aligned}
$$

Let $\kappa_{1}, \kappa_{2}$, and $\kappa_{3}$ be the roots of the cubic equation $\Pi(s)=0$.

Then, we have $\Pi(s)=\left(s-\kappa_{1}\right)\left(s-\kappa_{2}\right)\left(s-\kappa_{3}\right)$ and hence (52) gives

$$
m_{Z, j}^{(2) *}(s)=\frac{p_{3 j}(s)}{s\left(s-\omega_{1}\right)\left(s-\omega_{2}\right)\left(s-\omega_{3}\right)\left(s-\kappa_{1}\right)\left(s-\kappa_{2}\right)\left(s-\kappa_{3}\right)}, j=1,2,3 .
$$

Splitting into partial fractions, and then taking inverse Laplace transform, equation (60) gives

$$
\begin{aligned}
m_{Z, j}^{(2)}(t)=\frac{p_{3 j}(0)}{\omega_{1} \omega_{2} \omega_{3} \kappa_{1} \kappa_{2} \kappa_{3}}+\frac{p_{3 j}\left(\omega_{1}\right)}{\omega_{1}\left(\omega_{1}-\omega_{2}\right)\left(\omega_{1}-\omega_{3}\right)\left(\omega_{1}-\kappa_{1}\right)\left(\omega_{1}-\kappa_{2}\right)\left(\omega_{1}-\kappa_{3}\right)} e^{\omega_{1} t} & p_{3 j}\left(\omega_{2}\right) \\
& +\frac{p_{3 j}\left(\omega_{3}\right)}{\omega_{2}\left(\omega_{2}-\omega_{1}\right)\left(\omega_{2}-\omega_{3}\right)\left(\omega_{2}-\kappa_{1}\right)\left(\omega_{2}-\kappa_{2}\right)\left(\omega_{2}-\kappa_{3}\right)} e^{\omega_{2} t} \\
& +\frac{p_{3 j}\left(\kappa_{1}\right)}{\omega_{3}\left(\omega_{3}-\omega_{1}\right)\left(\omega_{3}-\omega_{2}\right)\left(\omega_{3}-\kappa_{1}\right)\left(\omega_{3}-\kappa_{2}\right)\left(\omega_{3}-\kappa_{3}\right)} e^{\omega_{3} t} \\
& +\frac{p_{3 j}\left(\kappa_{2}\right)}{\kappa_{1}\left(\kappa_{1}-\omega_{1}\right)\left(\kappa_{1}-\omega_{2}\right)\left(\kappa_{1}-\omega_{3}\right)\left(\kappa_{1}-\kappa_{2}\right)\left(\kappa_{1}-\kappa_{3}\right)} e^{\kappa_{1} t} \\
& +\frac{\kappa_{3 j}\left(\kappa_{3}\right)}{\left.\kappa_{2}-\omega_{1}\right)\left(\kappa_{2}-\omega_{2}\right)\left(\kappa_{2}-\omega_{3}\right)\left(\kappa_{2}-\kappa_{1}\right)\left(\kappa_{2}-\kappa_{3}\right)} e^{\kappa_{3} t}, j=1,2,3 .
\end{aligned}
$$

Taking Laplace transform on both sides of (10), (11) and (12), we get

$$
\begin{aligned}
& \left(s+a_{1}-2 L_{1}\right) m_{Y, 1}^{(2) *}(s)-\gamma_{12} m_{Y, 2}^{(2) *}(s)-\gamma_{13} m_{Y, 3}^{(2) *}(s)=0, \\
& -\gamma_{21} m_{Y, 1}^{(2) *}(s)+\left(s+a_{2}-L_{2}\right) m_{Y, 2}^{(2) *}(s)-\gamma_{23} m_{Y, 3}^{(2) *}(s)=L_{2} m_{Y, 2}^{(1) *}(s), \\
& -\gamma_{31} m_{Y, 1}^{(2) *}(s)-\gamma_{32} m_{Y, 2}^{(2) *}(s)+\left(s+a_{3}\right) m_{Y, 3}^{(2) *}(s)=2 L_{3} m_{Y, 3}^{(1) *}(s) .
\end{aligned}
$$

Solving (62),(63) and (64), we get

$$
m_{Y, 1}^{(2) *}(s)=\frac{\Pi_{21}(s)}{\Pi(s)}, m_{Y, 2}^{(2) *}(s)=\frac{\Pi_{22}(s)}{\Pi(s)}, m_{Y, 3}^{(2) *}(s)=\frac{\Pi_{23}(s)}{\Pi(s)},
$$

where, $\Pi(s)$ is same as (53) and 
International Journal of Mathematical, Engineering and Management Sciences

Vol. 5, No. 6, 1468-1487, 2020

https://doi.org/10.33889/IJMEMS.2020.5.6.109

$\Pi_{21}(s)=\left|\begin{array}{ccc}0 & -\gamma_{12} & -\gamma_{13} \\ L_{2} m_{Y, 2}^{(1) *}(s) & s+a_{2}-L_{2} & -\gamma_{23} \\ 2 L_{3} m_{Y, 3}^{(1) *}(s) & -\gamma_{32} & s+a_{3}\end{array}\right|$

$\Pi_{22}(s)=\left|\begin{array}{ccc}s+a_{1}-2 L_{1} & 0 & -\gamma_{13} \\ -\gamma_{21} & L_{2} m_{Y, 2}^{(1) *}(s) & -\gamma_{23} \\ -\gamma_{31} & 2 L_{3} m_{Y, 3}^{(1) *}(s) & s+a_{3}\end{array}\right|$,

$\Pi_{23}(s)=\left|\begin{array}{ccc}s+a_{1}-2 L_{1} & -\gamma_{12} & 0 \\ -\gamma_{21} & s+a_{2}-L_{2} & L_{2} m_{Y, 2}^{(1) *}(s) \\ -\gamma_{31} & -\gamma_{32} & 2 L_{3} m_{Y, 3}^{(1) *}(s)\end{array}\right|$.

Substituting (47) and (48) in (66), (67) and (68), and simplifying, we get

$\Pi_{21}(s)=\frac{q_{21}(s)}{\left(s-\omega_{1}\right)\left(s-\omega_{2}\right)\left(s-\omega_{3}\right)}$

$\Pi_{22}(s)=\frac{q_{22}(s)}{\left(s-\omega_{1}\right)\left(s-\omega_{2}\right)\left(s-\omega_{3}\right)}$,

$\Pi_{23}(s)=\frac{q_{23}(s)}{\left(s-\omega_{1}\right)\left(s-\omega_{2}\right)\left(s-\omega_{3}\right)}$,

where,

$$
\begin{aligned}
q_{21}(s)= & L_{2}\left[\gamma_{12}\left(s+a_{3}\right)+\gamma_{13} \gamma_{32}\right] \Delta_{22}(s)+2 L_{3}\left[\gamma_{12} \gamma_{23}+\gamma_{13}\left(s+a_{2}-L_{2}\right)\right] \Delta_{23}(s), \\
q_{22}(s)= & L_{2}\left[\left(s+a_{1}-2 L_{1}\right)\left(s+a_{3}\right)-\gamma_{13} \gamma_{31}\right] \Delta_{22}(s)+2 L_{3}\left[\left(s+a_{1}-2 L_{1}\right) \gamma_{23}+\right. \\
& \left.\gamma_{13} \gamma_{21}\right] \Delta_{23}(s), \\
q_{23}(s)= & L_{2}\left[\left(s+a_{1}-2 L_{1}\right) \gamma_{32}+\gamma_{12} \gamma_{31}\right] \Delta_{22}(s)+2 L_{3}\left[\left(s+a_{1}-2 L_{1}\right)\left(s+a_{2}-L_{2}\right)-\right. \\
& \left.\gamma_{12} \gamma_{21}\right] \Delta_{23}(s) .
\end{aligned}
$$

Since $\Pi(s)=\left(s-\kappa_{1}\right)\left(s-\kappa_{2}\right)\left(s-\kappa_{3}\right)$, equation (65) yields

$m_{Y, j}^{(2) *}(s)=\frac{q_{2 j}(s)}{\left(s-\omega_{1}\right)\left(s-\omega_{2}\right)\left(s-\omega_{3}\right)\left(s-\kappa_{1}\right)\left(s-\kappa_{2}\right)\left(s-\kappa_{3}\right)}, j=1,2,3$.

Splitting into partial fractions and then taking inverse Laplace transform, equation (72) yields

$$
\begin{aligned}
& m_{Y, j}^{(2)}(t)=\frac{q_{2 j}\left(\omega_{1}\right)}{\left(\omega_{1}-\omega_{2}\right)\left(\omega_{1}-\omega_{3}\right)\left(\omega_{1}-\kappa_{1}\right)\left(\omega_{1}-\kappa_{2}\right)\left(\omega_{1}-\kappa_{3}\right)} e^{\omega_{1} t} \\
& +\frac{q_{2 j}\left(\omega_{2}\right)}{\left(\omega_{2}-\omega_{1}\right)\left(\omega_{2}-\omega_{3}\right)\left(\omega_{2}-\kappa_{1}\right)\left(\omega_{2}-\kappa_{2}\right)\left(\omega_{2}-\kappa_{3}\right)} e^{\omega_{2} t} \\
& +\frac{q_{2 j}\left(\omega_{3}\right)}{\left(\omega_{3}-\omega_{1}\right)\left(\omega_{3}-\omega_{2}\right)\left(\omega_{3}-\kappa_{1}\right)\left(\omega_{3}-\kappa_{2}\right)\left(\omega_{3}-\kappa_{3}\right)} e^{\omega_{3} t}
\end{aligned}
$$


International Journal of Mathematical, Engineering and Management Sciences

Vol. 5, No. 6, 1468-1487, 2020

https://doi.org/10.33889/IJMEMS.2020.5.6.109

$$
\begin{gathered}
+\frac{q_{2 j}\left(\kappa_{1}\right)}{\left(\kappa_{1}-\omega_{1}\right)\left(\kappa_{1}-\omega_{2}\right)\left(\kappa_{1}-\omega_{3}\right)\left(\kappa_{1}-\kappa_{2}\right)\left(\kappa_{1}-\kappa_{3}\right)} e^{\kappa_{1} t} \\
+\frac{q_{2 j}\left(\kappa_{2}\right)}{\left(\kappa_{2}-\omega_{1}\right)\left(\kappa_{2}-\omega_{2}\right)\left(\kappa_{2}-\omega_{3}\right)\left(\kappa_{2}-\kappa_{1}\right)\left(\kappa_{2}-\kappa_{3}\right)} e^{\kappa_{2} t} \\
+\frac{q_{2 j}\left(\kappa_{3}\right)}{\left(\kappa_{3}-\omega_{1}\right)\left(\kappa_{3}-\omega_{2}\right)\left(\kappa_{3}-\omega_{3}\right)\left(\kappa_{3}-\kappa_{1}\right)\left(\kappa_{3}-\kappa_{2}\right)} e^{\kappa_{3} t}, j=1,2,3 .
\end{gathered}
$$

Taking Laplace transform on both sides of (7), (8) and (9), we get

$$
\begin{aligned}
& \left(s+a_{1}-2 L_{1}\right) m_{X, 1}^{(2) *}(s)-\gamma_{12} m_{X, 2}^{(2) *}(s)-\gamma_{13} m_{X, 3}^{(2) *}(s)=1, \\
& -\gamma_{21} m_{X, 1}^{(2) *}(s)+\left(s+a_{2}-L_{2}\right) m_{X, 2}^{(2) *}(s)-\gamma_{23} m_{X, 3}^{(2) *}(s)=1, \\
& -\gamma_{31} m_{X, 1}^{(2) *}(s)-\gamma_{32} m_{X, 2}^{(2) *}(s)+\left(s+a_{3}\right) m_{X, 3}^{(2) *}(s)=1 .
\end{aligned}
$$

Solving (74), (75) and (76), we get

$$
m_{X, 1}^{(2) *}(s)=\frac{\Pi_{11}(s)}{\Pi(s)}, m_{X, 2}^{(2) *}(s)=\frac{\Pi_{12}(s)}{\Pi(s)}, m_{X, 3}^{(2) *}(s)=\frac{\Pi_{13}(s)}{\Pi(s)},
$$

where, $\Pi(s)$ is same as $(53)$ and

$$
\begin{aligned}
& \Pi_{11}(s)=\left|\begin{array}{ccc}
1 & -\gamma_{12} & -\gamma_{13} \\
1 & s+a_{2}-L_{2} & -\gamma_{23} \\
1 & -\gamma_{32} & s+a_{3}
\end{array}\right|, \\
& \Pi_{12}(s)=\left|\begin{array}{ccc}
s+a_{1}-2 L_{1} & 1 & -\gamma_{13} \\
-\gamma_{21} & 1 & -\gamma_{23} \\
-\gamma_{31} & 1 & s+a_{3}
\end{array}\right|, \\
& \Pi_{13}(s)=\left|\begin{array}{ccc}
s+a_{1}-2 L_{1} & -\gamma_{12} & 1 \\
-\gamma_{21} & s+a_{2}-L_{2} & 1 \\
-\gamma_{31} & -\gamma_{32} & 1
\end{array}\right| .
\end{aligned}
$$

Since $\Pi(s)=\left(s-\kappa_{1}\right)\left(s-\kappa_{2}\right)\left(s-\kappa_{3}\right)$, equation (77) yields

$$
m_{X, j}^{(2) *}(s)=\frac{\Pi_{1 j}(s)}{\left(s-\kappa_{1}\right)\left(s-\kappa_{2}\right)\left(s-\kappa_{3}\right)}, j=1,2,3 .
$$

Splitting into partial fractions, and then taking inverse Laplace transform, equation (81) yields

$$
m_{X, j}^{(2)}(t)=\frac{\Pi_{1 j}\left(\kappa_{1}\right)}{\left(\kappa_{1}-\kappa_{2}\right)\left(\kappa_{1}-\kappa_{3}\right)} e^{\kappa_{1} t}+\frac{\Pi_{1 j}\left(\kappa_{2}\right)}{\left(\kappa_{2}-\kappa_{1}\right)\left(\kappa_{2}-\kappa_{3}\right)} e^{\kappa_{2} t}+\frac{\Pi_{1 j}\left(\kappa_{3}\right)}{\left(\kappa_{3}-\kappa_{1}\right)\left(\kappa_{3}-\kappa_{2}\right)} e^{\kappa_{3} t}, j=1,2,3 .
$$


International Journal of Mathematical, Engineering and Management Sciences

Vol. 5, No. 6, 1468-1487, 2020

https://doi.org/10.33889/IJMEMS.2020.5.6.109

\section{A Numerical Illustration}

For the purpose of illustration, we fix the values of the parameters based on Moolgavkar and Venzon (1979) as follows:

$$
\begin{array}{llllll}
\gamma_{12}=0.6 ; & \gamma_{13}=0.3 ; & \gamma_{21}=0.4 ; & \gamma_{23}=0.8 ; & \gamma_{31}=0.7 ; & \gamma_{32}=0.5 ; \\
L_{1}=0.3 ; & D_{1}=0.5 ; & L_{2}=0.4 ; & D_{2}=0.4 ; & L_{3}=0.5 ; & D_{3}=0.3 ; \\
\alpha_{1}=0.7 ; & \mu_{1}=0.5 ; & \alpha_{2}=0.6 ; & \mu_{2}=0.6 ; & \alpha_{3}=0.5 ; & \mu_{3}=0.7
\end{array}
$$

For the above values of the parameters, the zeros of $\Delta(s)$ are given by $\omega_{1}=-2.7618 ; \omega_{2}=$ $-1.868 ; \omega_{3}=-0.2702$ and the zeros of $\Pi(s)$ are given by $\kappa_{1}=-2.3708 ; \kappa_{2}=-1.9185 ; \kappa_{3}=$ -0.4107 . We have computed the mean number of malignant cells in the case of single mutation (see Table 1, Table 2 and Table 3), and depicted the graphs of $m_{Z, 1}^{(1)}(t), m_{Z, 2}^{(1)}(t)$, and $m_{Z, 3}^{(1)}(t)$ in Figure 2, Figure 3 and Figure 4. In all these three graphs, we find that the mean number of malignant cells increases in all the three levels of environment and it crosses the threshold value 1 as early as in 5.0 units of time in level 1, 7.0 units of time in level 2, and 9.0 units of time in level 3.

Table 1. Environment level 1 dependent growth of maligant cells (One mutation)

\begin{tabular}{|c|c|c|c|c|c|c|c|}
\hline$t$ & $m_{Z, 1}^{(1)}(t)$ & $t$ & $m_{Z, 1}^{(1)}(t)$ & $t$ & $m_{Z, 1}^{(1)}(t)$ & $t$ \\
\hline 0.5 & 0.0607 & 5.5 & 1.0384 & 10.5 & 1.3183 & 15.5 & 1.3908 \\
\hline 1.0 & 0.1834 & 6.0 & 1.0861 & 11.0 & 1.3306 & 16.0 & 1.3940 \\
\hline 1.5 & 0.3195 & 6.5 & 1.1278 & 11.5 & 1.3414 & 16.5 & 1.3968 \\
\hline 2.0 & 0.4502 & 7.0 & 1.1643 & 12.0 & 1.3509 & 17.0 & 1.3992 \\
\hline 2.5 & 0.5692 & 7.5 & 1.1961 & 12.5 & 1.3591 & 17.5 & 1.4013 \\
\hline 3.0 & 0.6750 & 8.0 & 1.2239 & 13.0 & 1.3663 & 18.0 & 1.4032 \\
\hline 3.5 & 0.7681 & 8.5 & 1.2482 & 13.5 & 1.3726 & 18.5 & 1.4048 \\
\hline 4.0 & 0.8498 & 9.0 & 1.2694 & 14.0 & 1.3781 & 19.0 & 1.4063 \\
\hline 4.5 & 0.9213 & 9.5 & 1.2879 & 14.5 & 1.3829 & 19.5 & 1.4075 \\
\hline 5.0 & 0.9838 & 10.0 & 1.3041 & 15.0 & 1.3871 & 20.0 & 1.4086 \\
\hline
\end{tabular}

Table 2. Environment level 2 dependent growth of maligant cells (One mutation)

\begin{tabular}{|c|c|c|c|c|c|c|c|}
\hline$t$ & $m_{Z, 2}^{(1)}(t)$ & $t$ & $m_{Z, 2}^{(1)}(t)$ & $t$ & $m_{Z, 2}^{(1)}(t)$ & $t$ \\
\hline 0.5 & 0.2560 & 5.5 & 0.9339 & 10.5 & 1.0722 & 15.5 & 1.1080 \\
\hline 1.0 & 0.4275 & 6.0 & 0.9575 & 11.0 & 1.0783 & 16.0 & 1.1096 \\
\hline 1.5 & 0.5439 & 6.5 & 0.9781 & 11.5 & 1.0837 & 16.5 & 1.1110 \\
\hline 2.0 & 0.6292 & 7.0 & 0.9961 & 12.0 & 1.0883 & 17.0 & 1.1122 \\
\hline 2.5 & 0.6965 & 7.5 & 1.0119 & 12.5 & 1.0924 & 17.5 & 1.1132 \\
\hline 3.0 & 0.7521 & 8.0 & 1.0256 & 13.0 & 1.0959 & 18.0 & 1.1142 \\
\hline 3.5 & 0.7995 & 8.5 & 1.0376 & 13.5 & 1.0991 & 18.5 & 1.1150 \\
\hline 4.0 & 0.8404 & 9.0 & 1.0481 & 14.0 & 1.1018 & 19.0 & 1.1157 \\
\hline 4.5 & 0.8760 & 9.5 & 1.0572 & 14.5 & 1.1041 & 19.5 & 1.1163 \\
\hline 5.0 & 0.9069 & 10.0 & 1.0652 & 15.0 & 1.1062 & 20.0 & 1.1168 \\
\hline
\end{tabular}


International Journal of Mathematical, Engineering and Management Sciences

Vol. 5, No. 6, 1468-1487, 2020

https://doi.org/10.33889/IJMEMS.2020.5.6.109

Table 3. Environment level 3 dependent growth of maligant cells (One mutation)

\begin{tabular}{|c|c|c|c|c|c|c|c|}
\hline$t$ & $m_{Z, 3}^{(1)}(t)$ & $t$ & $m_{Z, 3}^{(1)}(t)$ & $t$ & $m_{Z, 3}^{(1)}(t)$ & $t$ & $m_{Z, 3}^{(1)}(t)$ \\
\hline 0.5 & 0.3203 & 5.5 & 0.8952 & 10.5 & 1.0197 & 15.5 & 1.0519 \\
\hline 1.0 & 0.4675 & 6.0 & 0.9164 & 11.0 & 1.0252 & 16.0 & 1.0533 \\
\hline 1.5 & 0.5588 & 6.5 & 0.9350 & 11.5 & 1.0300 & 16.5 & 1.0546 \\
\hline 2.0 & 0.6276 & 7.0 & 0.9512 & 12.0 & 1.0341 & 17.0 & 1.0556 \\
\hline 2.5 & 0.6843 & 7.5 & 0.9653 & 12.5 & 1.0378 & 17.5 & 1.0566 \\
\hline 3.0 & 0.7327 & 8.0 & 0.9777 & 13.0 & 1.0410 & 18.0 & 1.0574 \\
\hline 3.5 & 0.7747 & 8.5 & 0.9885 & 13.5 & 1.0438 & 18.5 & 1.0581 \\
\hline 4.0 & 0.8112 & 9.0 & 0.9979 & 14.0 & 1.0463 & 19.0 & 1.0588 \\
\hline 4.5 & 0.8431 & 9.5 & 1.0062 & 14.5 & 1.0484 & 19.5 & 1.0593 \\
\hline 5.0 & 0.8709 & 10.0 & 1.0134 & 15.0 & 1.0503 & 20.0 & 1.0598 \\
\hline
\end{tabular}

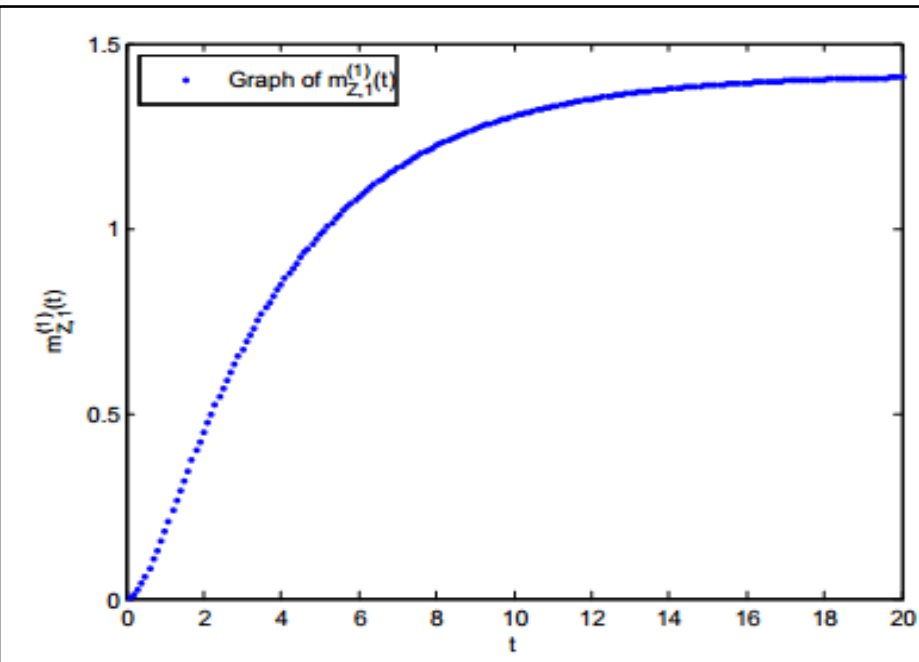

Figure 2. Graph of $m_{z, 1}^{(1)}(t)$

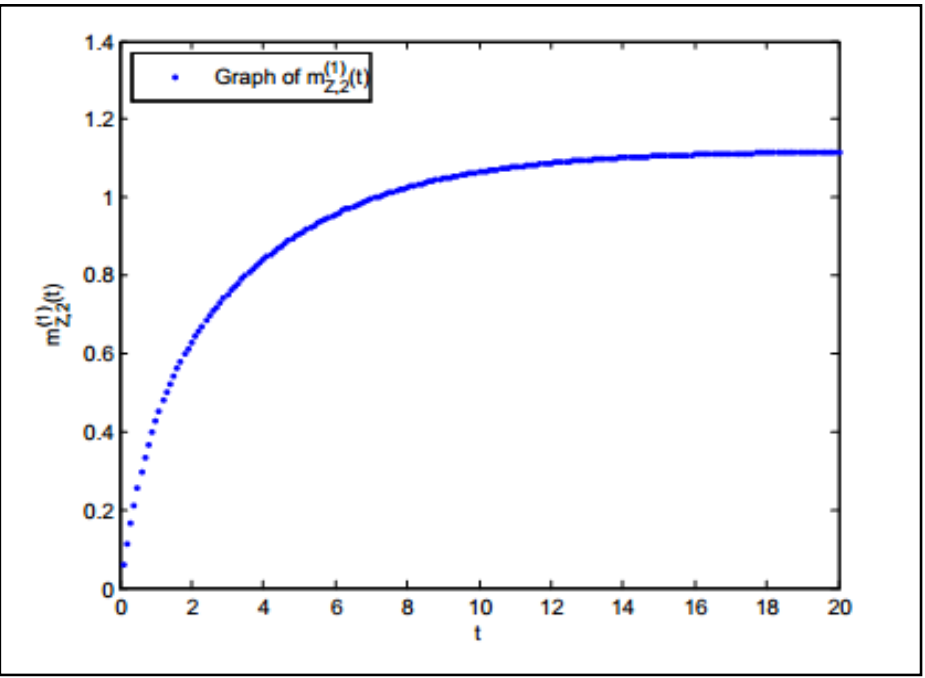

Figure 3. Graph of $m_{z, 2}^{(1)}(t)$ 
International Journal of Mathematical, Engineering and Management Sciences

Vol. 5, No. 6, 1468-1487, 2020

https://doi.org/10.33889/IJMEMS.2020.5.6.109

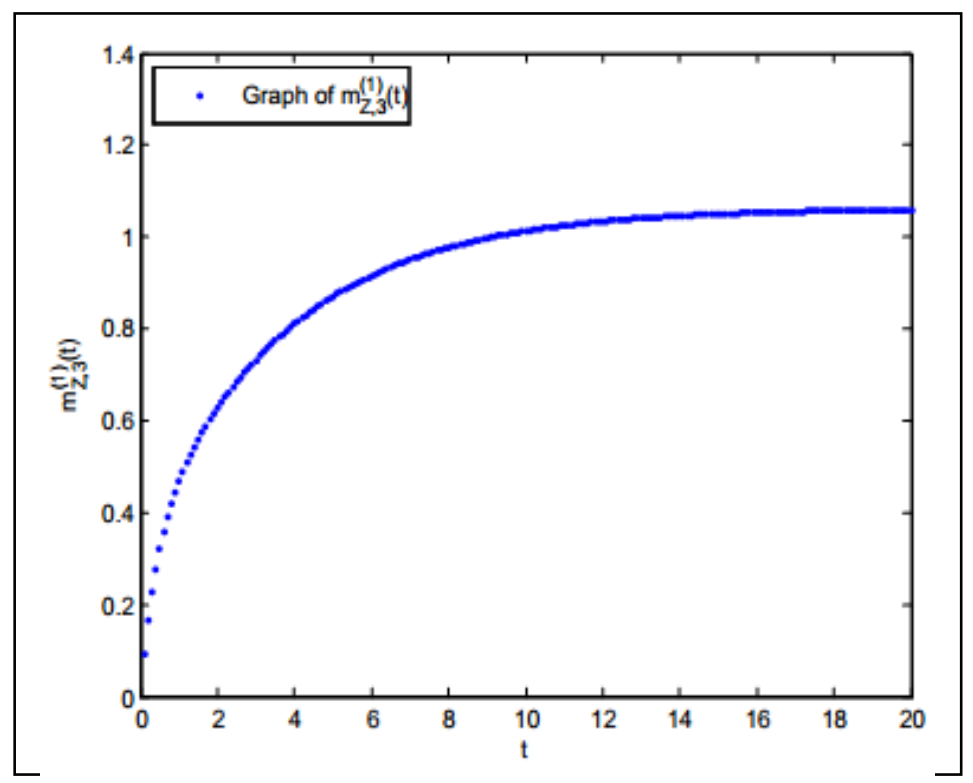

Figure 4. Graph of $m_{Z, 3}^{(1)}(t)$

For the same numerical values of the parameters, we have computed the mean number of malignant cells in the case of two mutation (see Table 4, table 5 and Table 6), and depicted the graphs of $m_{z, 1}^{(2)}(t), m_{z, 2}^{(2)}(t)$, and $m_{z, 3}^{(2)}(t)$ in Figure 5, Figure 6 and Figure 7 . In each of these graphs, we find that the mean number of malignant cells increases initially in level 1 of environment, but it has almost reached a steady state as early as in 42.1 units of time in level 1. On the other hand, the mean number of malignant cells increases for all time and crosses 1 in 13.7 units of time in level 2, and 10.5 units of time in level 3. In other words, environment level 3 induces the appearance of malignant cells in the population.

Table 4. Environment level 1 dependent growth of maligant cells (Two mutation)

\begin{tabular}{|c|c|c|c|c|c|c|c|}
\hline$t$ & $m_{Z, 1}^{(2)}(t)$ & $t$ & $m_{Z, 1}^{(2)}(t)$ & $t$ & $m_{Z, 1}^{(2)}(t)$ & $t$ & $m_{Z, 1}^{(2)}(t)$ \\
\hline 1.0 & 0.0334 & 11.0 & 0.7729 & 21.0 & 0.8631 & 31.0 & 0.8698 \\
\hline 2.0 & 0.1339 & 12.0 & 0.7947 & 22.0 & 0.8648 & 32.0 & 0.8699 \\
\hline 3.0 & 0.2527 & 13.0 & 0.8118 & 23.0 & 0.8661 & 33.0 & 0.8700 \\
\hline 4.0 & 0.3644 & 14.0 & 0.8251 & 24.0 & 0.8671 & 34.0 & 0.8701 \\
\hline 5.0 & 0.4617 & 15.0 & 0.8354 & 25.0 & 0.8678 & 35.0 & 0.8701 \\
\hline 6.0 & 0.5437 & 16.0 & 0.8434 & 26.0 & 0.8684 & 36.0 & 0.8701 \\
\hline 7.0 & 0.6112 & 17.0 & 0.8496 & 27.0 & 0.8689 & 37.0 & 0.8702 \\
\hline 8.0 & 0.6661 & 18.0 & 0.8544 & 28.0 & 0.8692 & 38.0 & 0.8702 \\
\hline 9.0 & 0.7101 & 19.0 & 0.8581 & 29.0 & 0.8695 & 39.0 & 0.8702 \\
\hline 10.0 & 0.7452 & 20.0 & 0.8609 & 30.0 & 0.8696 & 40.0 & 0.8702 \\
\hline
\end{tabular}


International Journal of Mathematical, Engineering and Management Sciences

Vol. 5, No. 6, 1468-1487, 2020

https://doi.org/10.33889/IJMEMS.2020.5.6.109

Table 5. Environment level 2 dependent growth of maligant cells (Two mutation)

\begin{tabular}{|c|c|c|c|c|c|c|c|}
\hline$t$ & $m_{z, 2}^{(2)}(t)$ & $t$ & $m_{z, 2}^{(2)}(t)$ & $t$ & $m_{Z, 2}^{(2)}(t)$ & $t$ & $m_{z, 2}^{(2)}(t)$ \\
\hline 0.5 & 0.0299 & 5.5 & 0.6843 & 10.5 & 0.9402 & 15.5 & 1.0174 \\
\hline 1.0 & 0.1006 & 6.0 & 0.7244 & 11.0 & 0.9529 & 16.0 & 1.0210 \\
\hline 1.5 & 0.1835 & 6.5 & 0.7604 & 11.5 & 0.9642 & 16.5 & 1.0242 \\
\hline 2.0 & 0.2654 & 7.0 & 0.7928 & 12.0 & 0.9741 & 17.0 & 1.0270 \\
\hline 2.5 & 0.3420 & 7.5 & 0.8218 & 12.5 & 0.9829 & 17.5 & 1.0294 \\
\hline 3.0 & 0.4127 & 8.0 & 0.8477 & 13.0 & 0.9906 & 18.0 & 1.0315 \\
\hline 3.5 & 0.4775 & 8.5 & 0.8708 & 13.5 & 0.9974 & 18.5 & 1.0334 \\
\hline 4.0 & 0.5367 & 9.0 & 0.8914 & 14.0 & 1.0034 & 19.0 & 1.0350 \\
\hline 4.5 & 0.5908 & 9.5 & 0.9096 & 14.5 & 1.0087 & 19.5 & 1.0365 \\
\hline 5.0 & 0.6398 & 10.0 & 0.9258 & 15.0 & 1.0134 & 20.0 & 1.0377 \\
\hline
\end{tabular}

Table 6. Environment level 3 dependent growth of maligant cells (Two mutation)

\begin{tabular}{|c|c|c|c|c|c|c|c|}
\hline$t$ & $m_{Z, 3}^{(2)}(t)$ & $t$ & $m_{Z, 3}^{(2)}(t)$ & $t$ & $m_{Z, 3}^{(2)}(t)$ & $t$ & $m_{Z, 3}^{(2)}(t)$ \\
\hline 0.5 & 0.0688 & 5.5 & 0.7548 & 10.5 & 0.9981 & 15.5 & 1.0706 \\
\hline 1.0 & 0.1713 & 6.0 & 0.7932 & 11.0 & 1.0101 & 16.0 & 1.0740 \\
\hline 1.5 & 0.2648 & 6.5 & 0.8277 & 11.5 & 1.0207 & 16.5 & 1.0769 \\
\hline 2.0 & 0.3480 & 7.0 & 0.8586 & 12.0 & 1.0300 & 17.0 & 1.0795 \\
\hline 2.5 & 0.4234 & 7.5 & 0.8861 & 12.5 & 1.0382 & 17.5 & 1.0818 \\
\hline 3.0 & 0.4922 & 8.0 & 0.9107 & 13.0 & 1.0455 & 18.0 & 1.0838 \\
\hline 3.5 & 0.5551 & 8.5 & 0.9326 & 13.5 & 1.0519 & 18.5 & 1.0855 \\
\hline 4.0 & 0.6125 & 9.0 & 0.9520 & 14.0 & 1.0575 & 19.0 & 1.0870 \\
\hline 4.5 & 0.6648 & 9.5 & 0.9693 & 14.5 & 1.0624 & 19.5 & 1.0884 \\
\hline 5.0 & 0.7121 & 10.0 & 0.9846 & 15.0 & 1.0668 & 20.0 & 1.0896 \\
\hline
\end{tabular}

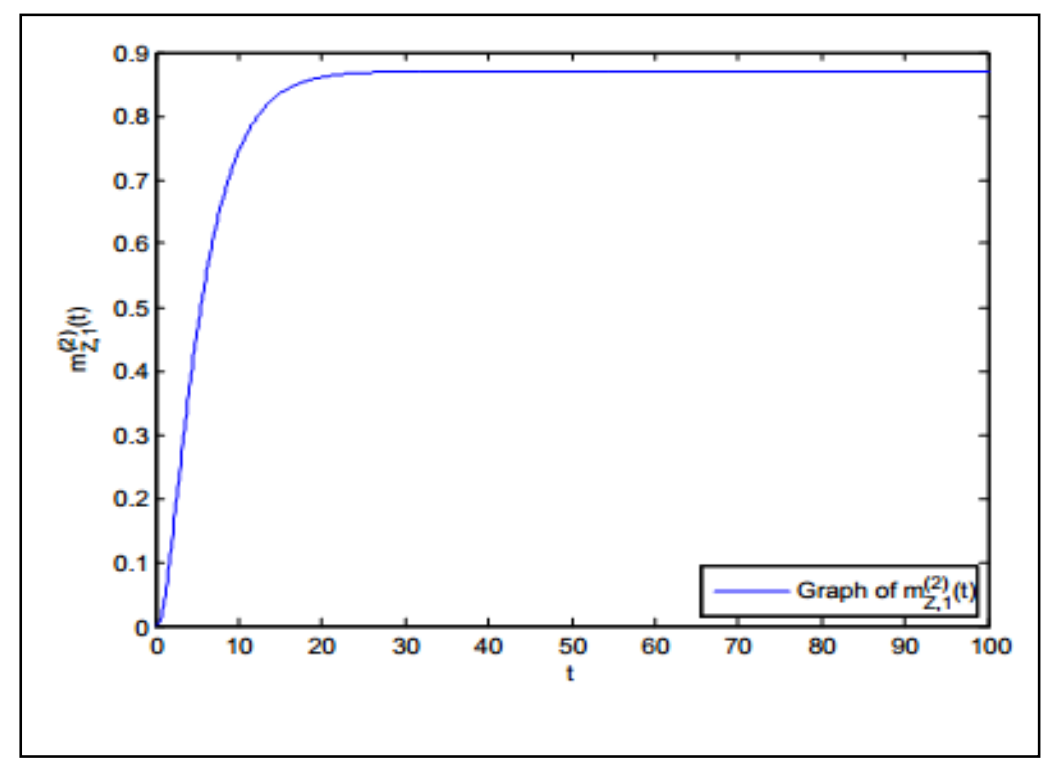

Figure 5. Graph of $m_{z, 1}^{(2)}(t)$ 
International Journal of Mathematical, Engineering and Management Sciences

Vol. 5, No. 6, 1468-1487, 2020

https://doi.org/10.33889/IJMEMS.2020.5.6.109

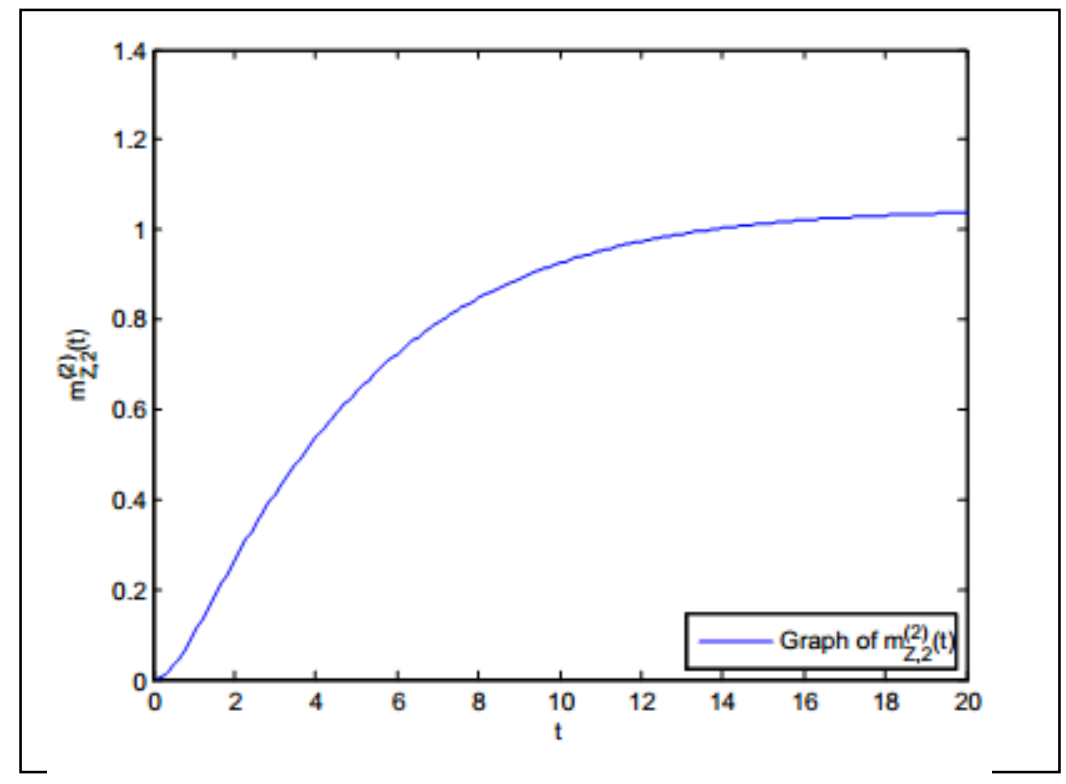

Figure 6. Graph of $m_{Z, 2}^{(2)}(t)$

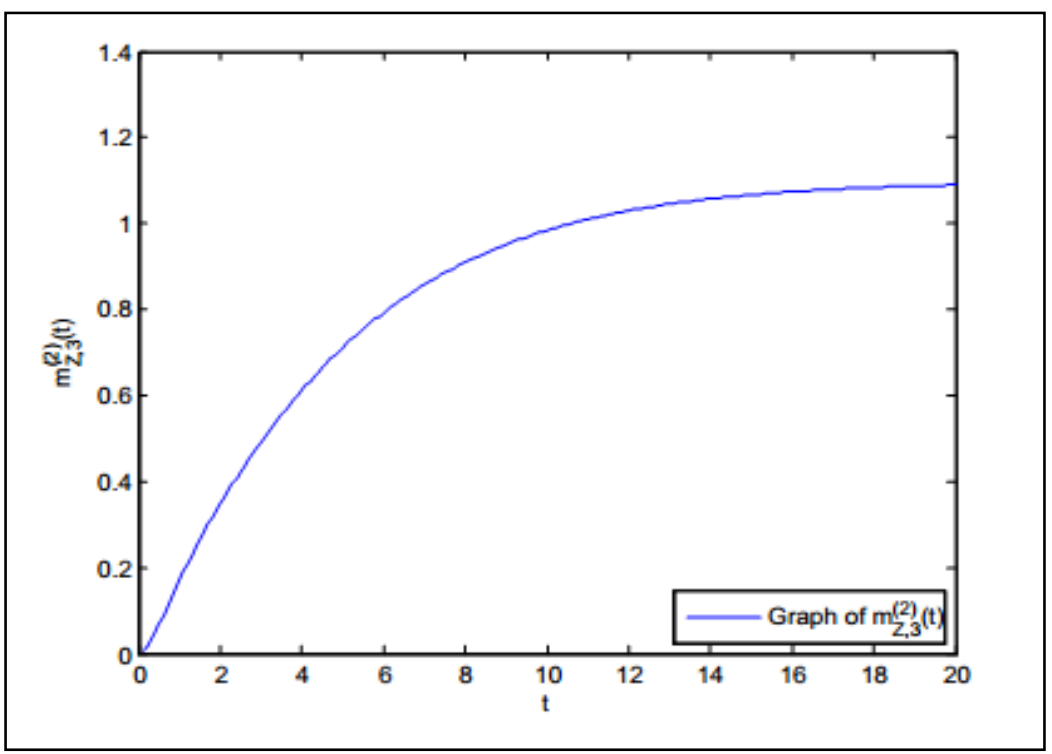

Figure 7. Graph of $m_{Z, 3}^{(2)}(t)$

We present now a comparison between the results of the present model on three-level environment with the results of Yadavalli et al. (2020) on two-level environment. When there are only two levels for environmental changes, the onset of malignant cells for one mutation model is quicker (between 1.9- 2.0 units of time) than when there are three level for environmental changes (between 5.1-5.2 units of time). We also find that when there are only two levels for 
International Journal of Mathematical, Engineering and Management Sciences

Vol. 5, No. 6, 1468-1487, 2020

https://doi.org/10.33889/IJMEMS.2020.5.6.109

environmental changes, the onset of malignant cells for two mutation model is quicker (between $1.9-2.0$ units of time) than when there are three level for environmental changes (between $13.7-13.8$ units of time). This is because of the fact that when the favourable conditions for mutation are available in a higher level, the transitions from one level to another level of the environment affect the time of progression of malignant cells.

\section{Conclusion}

We proposed an environment dependent two mutation model for carcinogenesis. We assumed three levels for the environment in which the third level is favourable for mutation into malignant cells. Based upon the numerical illustration, we are able to conclude that two mutation model shows that malignant cells occur delayed in time compared to the single mutation model. Further more, it is observed that environment level 3 is favourable for the production of malignant cells.

\section{Conflict of Interest}

The authors have equal contribution in this work and it is declared that there is no conflict of interest for this publication.

\section{Acknowledgment}

The authors express their sincere gratitude to the referees for their valuable suggestions towards the improvement of the paper.

\section{References}

Armitage, P., \& Doll, R. (1954). The age distribution of cancer and a multi-stage theory of carcinogenesis. British Journal of Cancer, 8(1), 1-12.

Armitage, P., \& Doll, R. (1957). A two-stage theory of carcinogenesis in relation to the age distribution of human cancer. British Journal of Cancer, 11(2), 161-169.

Armitage, P., \& Doll, R. (1961). Stochastic models for carcinogenesis. In Proceedings of the Fourth Berkeley Symposium on Mathematical Statistics and Probability, 4(30), 9-38.

Bellman, R., Kalaba, R., \& Wing, G.M. (1960). Invariant imbedding and mathematical physics. I. particle processes. Journal of Mathematical Physics, 1(4), 280-308.

Hochberg, M.E., \& Noble, R.J. (2017). A framework for how environment contributes to cancer risk. Ecology Letters, 20(2), 117-134.

Knudson, A.G. (1971). Mutation and cancer: statistical study of retinoblastoma. Proceedings of the National Academy of Sciences, 68(4), 820-823.

Martincorena, I., Raine, K.M., Gerstung, M., Dawson, K.J., Haase, K., Loo, P.V., Davies, H., Stratton, M.R., \& Campbell, P.J. (2017). Universal patterns of selection in cancer and somatic tissues. Cell,171(5), 1029-1041.

Moolgavkar, S.H., \& Knudson, A.G. (1981). Mutation and cancer: a model for human carcinogenesis. Journal of the National Cancer Institute, 66(6), 1037-1052.

Moolgavkar, S.H., \& Venzon, D.J. (1979). Two-event models for carcinogenesis: incidence curves for childhood and adult tumors. Mathematical Biosciences, 47(1-2), 55-77.

Nordling, C.O. (1953). A new theory on the cancer-inducing mechanism. British Journal of Cancer, 7(1), 68-72. 
International Journal of Mathematical, Engineering and Management Sciences

Vol. 5, No. 6, 1468-1487, 2020

https://doi.org/10.33889/IJMEMS.2020.5.6.109

Reddy, B.Y., Miller, D.M., \& Tsao, H. (2017). Somatic driver mutations in melanoma. Cancer, 123, 21042117.

Rozhok, A.I., \& DeGregori, J. (2016). The evolution of lifespan and age-dependent cancer risk. Trends in Cancer, 2(10), 552-560.

Rozhok, A.I., \& DeGregori, J. (2019). A generalized theory of age-dependent carcinogenesis. Elife, 8, e39950. Doi: 10.7554/eLife.39950.001.

Rozhok, A.I., Salstrom, J.L., \& DeGregori, J. (2016). Stochastic modeling reveals an evolutionary mechanism underlying elevated rates of childhood leukemia. Proceedings of the National Academy of Sciences, 113(4), 1050-1055.

Sun, S., Klebaner, F., \& Tian, T. (2014). A new model of time scheme for progression of colorectal cancer. BMC Systems Biology, 8(S3), S2. Doi: 10.1186/1752-0509-8-S3-S2.

Tomasetti, C., \& Vogelstein, B. (2015). Variation in cancer risk among tissues can be explained by the number of stem cell divisions. Science, 347(6217), 78-81.

Wolf, D.C., Cohen, S.M., Boobis, A.R., Dellarco, V.L., Fenner-Crisp, P.A., Moretto, A., Pastoor, T.P., Schoeny, R.S., Seed, J.G., \& Doe, J.E. (2019). Chemical carcinogenicity revisited 1: a unified theory of carcinogenicity based on contemporary knowledge. Regulatory Toxicology and Pharmacology, 103, 8692.

Yadavalli, V.S.S., Udayabaskaran, S., Dora Pravina, C.T., \& Sreelakshmi, S. (2020). A two-stage mutation stochastic model of carcinogenesis driven by a two level random environment. International Journal of Mathematical, Engineering and Management Sciences, 5(6), 1392-1408. 